PROCEEDINGS OF THE

AMERICAN MATHEMATICAL SOCIETY

Volume 138, Number 7, July 2010, Pages 2585-2590

S 0002-9939(10)10280-9

Article electronically published on February 26, 2010

\title{
ON THE NUMBER OF UNIVERSAL SOFIC GROUPS
}

\author{
SIMON THOMAS
}

(Communicated by Julia Knight)

\begin{abstract}
If $C H$ fails, then there exist $2^{2^{\aleph_{0}}}$ universal sofic groups up to isomorphism.
\end{abstract}

\section{INTRODUCTION}

Let $\mathcal{U}$ be a nonprincipal ultrafilter over $\omega$ and let $G_{\mathcal{U}}=\prod_{\mathcal{U}} \operatorname{Sym}(n)$ be the corresponding ultraproduct of the finite symmetric groups. Then Allsup-Kaye [1] and Elek-Szabó [2] have independently shown that $G_{\mathcal{U}}$ has a unique maximal proper normal subgroup; namely,

$$
M_{\mathcal{U}}=\left\{\left(\pi_{n}\right)_{\mathcal{U}} \in G_{\mathcal{U}}: \lim _{\mathcal{U}} \frac{\left|\operatorname{supp}\left(\pi_{n}\right)\right|}{n}=0\right\},
$$

where $\operatorname{supp}\left(\pi_{n}\right)=\left\{\ell \in n: \pi_{n}(\ell) \neq \ell\right\}$. Let $S_{\mathcal{U}}=G_{\mathcal{U}} / M_{\mathcal{U}}$. Then by Elek-Szabó [2], if $\Gamma$ is a finitely generated group, the following statements are equivalent:

- $\Gamma$ is a sofic group.

- $\Gamma$ embeds into $S_{\mathcal{U}}$ for some (equivalently every) nonprincipal ultrafilter $\mathcal{U}$. For this reason, $S_{\mathcal{U}}$ is said to be a universal sofic group 1 Of course, if $\mathcal{U} \neq \mathcal{D}$ are distinct nonprincipal ultrafilters over $\omega$, then there is no reason to expect that $S_{\mathcal{U}}$ and $S_{\mathcal{D}}$ will be isomorphic. In this paper, we will consider the problem of computing the number of universal sofic groups $S_{\mathcal{U}}$ up to isomorphism. Perhaps surprisingly, this problem turns out to be much easier to handle under the assumption that the Continuum Hypothesis $\mathrm{CH}$ fails.

Theorem 1.1. If $C H$ fails, then there exist $2^{2^{\aleph_{0}}}$ universal sofic groups $S_{\mathcal{U}}$ up to isomorphism.

On the other hand, suppose that $C H$ holds. Then each ultraproduct $G_{\mathcal{U}}=$ $\prod_{\mathcal{U}} \operatorname{Sym}(n)$ is saturated and hence is determined up to isomorphism by its first order theory. Thus there are at most $2^{\aleph_{0}}$ such ultraproducts up to isomorphism and hence at most $2^{\aleph_{0}}$ universal sofic groups up to isomorphism. It is easily shown that (as expected) there are $2^{\aleph_{0}}$ such ultraproducts up to elementary equivalence.

Received by the editors August 15, 2009, and, in revised form, November 6, 2009.

2010 Mathematics Subject Classification. Primary 03C20, 03E35, $20 \mathrm{~F} 69$.

Key words and phrases. Ultraproducts, expander graphs, sofic groups.

Research partially supported by NSF Grant DMS 0600940.

${ }^{1} \mathrm{~A}$ clear account of the basic theory of sofic groups can be found in Pestov [9]. It is an important open problem whether every finitely generated group is sofic.

(C)2010 American Mathematical Society Reverts to public domain 28 years from publication 
However, it is currently not even known whether there exist two nonisomorphic universal sofic groups if $\mathrm{CH}$ holds.

Conjecture 1.2. If $C H$ holds, then there exist $2^{\aleph_{0}}$ universal sofic groups $S_{\mathcal{U}}$ up to isomorphism.

Question 1.3. Are all universal sofic groups $S_{\mathcal{U}}$ elementarily equivalent?

It would be very interesting to know whether the results in this paper can be extended to the setting of hyperlinear groups 2 (At first glance, the techniques in this paper do not seem to generalize readily from finite symmetric groups to finite rank unitary groups.) In this context, the referee has pointed out that the results of Ge-Hadwin [4] suggest that if $\mathrm{CH}$ holds, then perhaps all metric ultraproducts of the finite rank unitary groups are isomorphic; i.e., if $\mathrm{CH}$ holds, then perhaps there exists a unique universal hyperlinear group up to isomorphism.

This paper is organized as follows. In Section 2 we will use a basic property of expander graphs to show that certain ultraproducts $\prod_{\mathcal{D}} G_{n}$ of finite groups can be realized as centralizers of finitely generated subgroups in suitably chosen universal sofic groups. Then in Section [3, using recent results of Kassabov [5] and EllisHachtman-Schneider-Thomas [3], we will complete the proof of Theorem 1.1

\section{Centralizers And EXPANDER families}

In this section, we will use a basic property of expander graphs to show that certain ultraproducts $\prod_{\mathcal{D}} G_{n}$ of finite groups can be realized as centralizers of finitely generated subgroups in suitably chosen universal sofic groups. We will begin by defining the notion of an expander family of finite graphs. Suppose that $\Gamma=(V, E)$ is a finite connected graph with vertex set $V$ and edge set $E$. Then for each subset $A \subseteq V$, the corresponding edge boundary is defined to be

$$
\partial A=\{e \in E:|e \cap A|=1\},
$$

and the expansion constant of $\Gamma$ is defined to be

$$
h(\Gamma)=\min \left\{\frac{|\partial A|}{|A|}: A \subset V \text { with } 1 \leq|A| \leq \frac{|V|}{2}\right\} .
$$

From now on, we will identify each finite graph $\Gamma=(V, E)$ with its vertex set $V$ and we will write $h(V)$ instead of $h(\Gamma)$. A finite graph $V$ is said to be $k$-regular if each vertex $v \in V$ has degree $k$.

Definition 2.1. Let $\left(V_{n}: n \in \omega\right.$ ) be a family of finite connected $k$-regular graphs such that $\left|V_{m}\right|<\left|V_{n}\right|$ for all $m<n \in \omega$. Then $\left(V_{n}: n \in \omega\right)$ is said to be an expander family if there exists $\tau>0$ such that $h\left(V_{n}\right) \geq \tau$ for all $n \in \omega$.

Most of the known expander families consist of suitably chosen Cayley graphs of finite groups. (For example, see Lubotzky [8] and Kassabov-Lubotzky-Nikolov [6].) Here if $G$ is a finite group and $S \subseteq G \backslash 1$ is a generating set, then the corresponding Cayley graph Cay $(G, S)$ is the graph with vertex set $G$ and edge set

$$
E=\left\{\{x, y\} \mid y=s x \text { for some } s \in S \cup S^{-1}\right\} .
$$

As we will see in Section 3 , Theorem 1.1 is an easy consequence of the following theorem, together with recent results of Kassabov [5] and Ellis-Hachtman-SchneiderThomas [3].

\footnotetext{
${ }^{2}$ A clear account of the basic theory of hyperlinear groups can also be found in Pestov $\underline{9}$.
} 
Theorem 2.2. For each $n \in \omega$, let $G_{n}$ be a finite group and let $S_{n} \subseteq G_{n}$ be a generating set of fixed size d. If $\left(\operatorname{Cay}\left(G_{n}, S_{n}\right): n \in \omega\right)$ is an expander family, then for each nonprincipal ultrafilter $\mathcal{D}$ over $\omega$, there exists a nonprincipal ultrafilter $\mathcal{U}$ over $\omega$ and a finitely generated subgroup $\Gamma \leqslant S_{\mathcal{U}}$ such that $C_{S_{\mathcal{U}}}(\Gamma) \cong \prod_{\mathcal{D}} G_{n}$.

The proof of Theorem 2.2 makes use of the following observation.

Proposition 2.3. Suppose that $V$ is a finite connected $k$-regular graph and that $h(V) \geq \tau$. Suppose that $\varepsilon>0$ and let $\delta=\varepsilon \tau /(\tau+k)$. Then whenever $Y \subseteq V$ is a subgraph with $|Y| \geq(1-\delta)|V|$, there exists a connected subgraph $Z \subseteq Y$ with $|Z| \geq(1-\varepsilon)|V|$.

Proof. Let $Y \subseteq V$ be a subgraph with $|Y| \geq(1-\delta)|V|$ and suppose that $C_{1}, \cdots, C_{t}$ are connected components of $Y$ with $\left|C_{i}\right| \leq \frac{1}{2}|V|$ for each $1 \leq i \leq t$. Consider the set

$$
P=\left\{(v, e): e \in \bigcup_{i=1}^{t} \partial C_{i} \text { and } v \in e \backslash Y\right\} .
$$

Notice that if $e \in \bigcup_{i=1}^{t} \partial C_{i}$, then $|e \cap Y|=1$. Thus

$$
|P|=\sum_{i=1}^{t}\left|\partial C_{i}\right| \geq \tau \sum_{i=1}^{t}\left|C_{i}\right|
$$

Clearly we also have that

$$
|P| \leq k|V \backslash Y| \leq k \delta|V|
$$

Hence we have that

$$
\tau \sum_{i=1}^{t}\left|C_{i}\right| \leq k \delta|V|
$$

and so

$$
|V \backslash Y|+\sum_{i=1}^{t}\left|C_{i}\right| \leq \delta|V|+\frac{k \delta}{\tau}|V|=\varepsilon|V| .
$$

It follows that $Y$ has a connected component $Z$ with $|Z| \geq(1-\varepsilon)|V|$.

The proof of Theorem 1.1 also makes use of the notions of the left regular and right regular permutation representations of a finite group $G$. Here the left regular permutation representation of $G$ is the embedding $\lambda: G \rightarrow \operatorname{Sym}(G)$ defined by $\lambda(g)(x)=g x$, and the right regular permutation representation of $G$ is the embedding $\rho: G \rightarrow \operatorname{Sym}(G)$ defined by $\rho(g)=x g^{-1}$. It is well-known that

$$
C_{\mathrm{Sym}(G)}(\lambda[G])=\rho[G] .
$$

(For example, see Tsuzuku [10, Theorem 3.2.10].)

Proof of Theorem 2.2. To simplify notation, suppose that $d=2$ and let $S_{n}=$ $\left\{a_{n}, b_{n}\right\}$. Let $\mathcal{U}$ be the nonprincipal ultrafilter over $\omega$ such that for each $X \subseteq \omega$,

$$
\left\{\left|G_{n}\right|: n \in X\right\} \in \mathcal{U} \quad \Longleftrightarrow \quad X \in \mathcal{D} .
$$

Then we can define a natural isomorphism

$$
\begin{aligned}
\sigma: \prod_{\mathcal{D}} \operatorname{Sym}\left(\left|G_{n}\right|\right) & \rightarrow \prod_{\mathcal{U}} \operatorname{Sym}(n) \\
\left(\theta_{n}\right)_{\mathcal{D}} & \mapsto\left(\psi_{n}\right)_{\mathcal{U}}
\end{aligned}
$$


by setting

$$
\psi_{n}= \begin{cases}\theta_{m} & \text { if } n=\left|G_{m}\right| \\ 1 & \text { otherwise. }\end{cases}
$$

Clearly there also exists a natural isomorphism $\iota: \prod_{\mathcal{D}} \operatorname{Sym}\left(G_{n}\right) \rightarrow \prod_{\mathcal{D}} \operatorname{Sym}\left(\left|G_{n}\right|\right)$. Let $\pi: \prod_{\mathcal{D}} \operatorname{Sym}\left(G_{n}\right) \rightarrow S_{\mathcal{U}}$ be the surjective homomorphism obtained by composing the following maps:

$$
\prod_{\mathcal{D}} \operatorname{Sym}\left(G_{n}\right) \stackrel{\iota}{\rightarrow} \prod_{\mathcal{D}} \operatorname{Sym}\left(\left|G_{n}\right|\right) \stackrel{\sigma}{\rightarrow} \prod_{\mathcal{U}} \operatorname{Sym}(n) \rightarrow\left(\prod_{\mathcal{U}} \operatorname{Sym}(n)\right) / M_{\mathcal{U}}=S_{\mathcal{U}} .
$$

For each $n \in \omega$, let $\lambda_{n}: G_{n} \rightarrow \operatorname{Sym}\left(G_{n}\right)$ and $\rho_{n}: G_{n} \rightarrow \operatorname{Sym}\left(G_{n}\right)$ be the left regular and right regular permutation representations. Let $\alpha, \beta \in S_{\mathcal{U}}$ be the elements defined by

$$
\alpha=\pi\left(\left(\lambda_{n}\left(a_{n}\right)\right)_{\mathcal{D}}\right) \quad \text { and } \quad \beta=\pi\left(\left(\lambda_{n}\left(b_{n}\right)\right)_{\mathcal{D}}\right) .
$$

Then we claim that the subgroup $\Gamma=\langle\alpha, \beta\rangle$ of $S_{\mathcal{U}}$ satisfies our requirements. Let $G=\prod_{\mathcal{D}} \operatorname{Sym}\left(G_{n}\right)$. Then clearly we have that

$$
\prod_{\mathcal{D}} \rho_{n}\left[G_{n}\right] \leqslant C_{G}\left(\left\{\left(\lambda_{n}\left(a_{n}\right)\right)_{\mathcal{D}},\left(\lambda_{n}\left(b_{n}\right)\right)_{\mathcal{D}}\right\}\right) .
$$

(In fact, using Tsuzuku [10, Theorem 3.2.10], it is easily seen that the two groups in the above inclusion are actually equal.) It is also clear that $\pi$ maps $\prod_{\mathcal{D}} \rho_{n}\left[G_{n}\right]$ injectively into $C_{S_{\mathcal{U}}}(\Gamma)$. Thus it is enough to show that if $\gamma \in C_{S_{\mathcal{U}}}(\Gamma)$, then there exists $g \in \prod_{\mathcal{D}} \rho_{n}\left[G_{n}\right]$ such that $\pi(g)=\gamma$. To see this, let $\varphi=\left(\varphi_{n}\right)_{\mathcal{D}}$ be an element such that $\pi(\varphi)=\gamma$ and fix some $0<\varepsilon<1 / 3$. Since $\left(\operatorname{Cay}\left(G_{n}, S_{n}\right): n \in \omega\right)$ is an expander family, Proposition 2.3 implies that there exists $\delta>0$ such that for all $n \in \omega$, if $Y \subseteq G_{n}$ is a subgraph of $\operatorname{Cay}\left(G_{n}, S_{n}\right)$ with $|Y| \geq(1-\delta)\left|G_{n}\right|$, then there exists a connected subgraph $Z \subseteq Y$ with $|Z| \geq(1-\varepsilon)\left|G_{n}\right|$. For each $n \in \omega$, let $Y_{n} \subseteq G_{n}$ be the set of elements $y \in G_{n}$ such that

$$
s \varphi_{n}(y)=\varphi_{n}(s y) \quad \text { for all } s \in S_{n} \cup S_{n}^{-1} .
$$

Then $A_{\varepsilon}=\left\{n \in \omega:\left|Y_{n}\right| \geq(1-\delta)\left|G_{n}\right|\right\} \in \mathcal{D}$. Fix some $n \in A_{\varepsilon}$. Then regarding $Y_{n}$ as a subgraph of the Cayley graph Cay $\left(G_{n}, S_{n}\right)$, there exists a connected subgraph $Z_{n} \subseteq Y_{n}$ such that $\left|Z_{n}\right| \geq(1-\varepsilon)\left|G_{n}\right|$. Fix some $z_{n} \in Z_{n}$ and let $\varphi_{n}\left(z_{n}\right)=z_{n} g_{n}$. Then applying (2.1) repeatedly, we obtain that $\varphi_{n}(z)=z g_{n}$ for all $z \in Z_{n}$. Note that if $g_{n}^{\prime} \in G_{n}$ with $g_{n}^{\prime} \neq g_{n}$, then $x g_{n}^{\prime} \neq x g_{n}$ for all $x \in G_{n}$. Hence if $0<\varepsilon^{\prime}<1 / 3$ and $n \in A_{\varepsilon^{\prime}} \cap A_{\varepsilon}$, then the above argument will yield precisely the same element $g_{n} \in G_{n}$. Hence, letting $g_{n}=1$ for $n \notin A_{\varepsilon}$, it follows that

$$
\pi\left(\left(\rho_{n}\left(g_{n}^{-1}\right)\right)_{\mathcal{D}}\right)=\pi\left(\left(\varphi_{n}\right)_{\mathcal{D}}\right)=\gamma
$$

as required.

\section{Ultraproducts of Finite ALternating GROUPS}

In this section, we will complete the proof of Theorem 1.1. We will make use of the following recent result of Kassabov [5].

Theorem 3.1. For each $n \geq 5$, there exists a generating subset $S_{n} \subseteq \operatorname{Alt}(n)$ with $\left|S_{n}\right|=20$ such that $\left(\operatorname{Cay}\left(\operatorname{Alt}(n), S_{n}\right): n \geq 5\right)$ is an expander family.

The following result is an immediate consequence of Theorems 2.2 and 3.1 . 
Theorem 3.2. For each nonprincipal ultrafilter $\mathcal{D}$ over $\omega$, there exist a nonprincipal ultrafilter $\mathcal{U}$ over $\omega$ and a finitely generated subgroup $\Gamma \leqslant S_{\mathcal{U}}$ such that $C_{S_{\mathcal{U}}}(\Gamma) \cong \prod_{\mathcal{D}} \operatorname{Alt}(n)$.

We will also make use of the following recent result of Ellis-Hachtman-SchneiderThomas [3].

Theorem 3.3. If $C H$ fails, then there exist $2^{2^{\aleph_{0}}}$ ultraproducts $\prod_{\mathcal{U}} \operatorname{Alt}(n)$ up to isomorphism.

Sketch Proof. By Allsup-Kaye [1, if $\mathcal{U}$ is a nonprincipal ultrafilter over $\omega$, then there is an inclusion-preserving bijection between the collection of proper normal subgroups of $\prod_{\mathcal{U}} \operatorname{Alt}(n)$ and the linearly ordered set

$$
L_{\mathcal{U}}=\left\{I \subseteq \prod_{\mathcal{U}} n: I \text { is an additive cut of } \prod_{\mathcal{U}} \mathbb{N}\right\}
$$

where an additive cut is a nonempty initial segment of the nonstandard model of arithmetic $\mathcal{M}=\prod_{\mathcal{U}} \mathbb{N}$ which is closed under addition in $\mathcal{M}$. A routine modification of the proof of Kramer-Shelah-Tent-Thomas [7, Theorem 3.3] shows that if $C H$ fails, then there exist $2^{2^{\aleph_{0}}}$ such linearly ordered sets $L_{\mathcal{U}}$ up to isomorphism.

Proof of Theorem 1.1, Let $\left\{\mathcal{D}_{\alpha}: \alpha<2^{2^{\aleph_{0}}}\right\}$ be a collection of nonprincipal ultrafilters over $\omega$ such that the corresponding ultraproducts $\prod_{\mathcal{D}_{\alpha}} \operatorname{Alt}(n)$ are pairwise nonisomorphic. Then for each $\alpha<2^{2^{\aleph_{0}}}$, there exist a nonprincipal ultrafilter $\mathcal{U}_{\alpha}$ over $\omega$ and a finitely generated subgroup $\Gamma_{\alpha} \leqslant S_{\mathcal{U}_{\alpha}}$ such that $C_{S_{\mathcal{U}_{\alpha}}}\left(\Gamma_{\alpha}\right) \cong \prod_{\mathcal{D}_{\alpha}} \operatorname{Alt}(n)$. Fix some $\alpha<2^{2^{\aleph_{0}}}$. Since $\left|S_{\mathcal{U}_{\alpha}}\right|=2^{\aleph_{0}}$, it follows that $S_{\mathcal{U}_{\alpha}}$ has only $2^{\aleph_{0}}$ finitely generated subgroups and hence there exist at most $2^{\aleph_{0}}$ ordinals $\beta<2^{2^{\aleph_{0}}}$ such that $S_{\mathcal{U}_{\alpha}} \cong S_{\mathcal{U}_{\beta}}$. It follows that $\left\{S_{\mathcal{U}_{\alpha}}: \alpha<2^{2^{\aleph_{0}}}\right\}$ includes a collection of $2^{2^{\aleph_{0}}}$ pairwise nonisomorphic groups.

\section{REFERENCES}

[1] J. Allsup and R. Kaye, Normal subgroups of nonstandard symmetric and alternating groups, Arch. Math. Logic 46 (2007), 107-121. MR.2298607 (2007k:03098)

[2] G. Elek and E. Szabó, Hyperlinearity, essentially free actions and $L^{2}$-invariants. The sofic property, Math. Ann. 332 (2005), 421-441. MR2178069 (2007i:43002)

[3] P. Ellis, S. Hachtman, S. Schneider and S. Thomas, Ultraproducts of finite alternating groups, RIMS Kokyuroku, No. 1619 (2008), 1-7.

[4] L. Ge and D. Hadwin, Ultraproducts of $C^{*}$-algebras, Recent advances in operator theory and related topics (Szeged, 1999), Oper. Theory Adv. Appl., vol. 127, Birkhäuser, Basel, 2001, pp. 305-326. MR.1902808 (2003f:46083)

[5] M. Kassabov, Symmetric groups and expander graphs, Invent. Math. 170 (2007), 327-354. MR2342639 (2008g:20009)

[6] M. Kassabov, A. Lubotzky and N. Nikolov, Finite simple groups as expanders, Proc. Natl. Acad. Sci. USA 103 (2006), 6116-6119. MR2221038 (2007d:20025)

[7] L. Kramer, S. Shelah, K. Tent and S. Thomas, Asymptotic cones of finitely presented groups, Adv. Math. 193 (2005), 142-173. MR2132762 (2006d:20075)

[8] A. Lubotzky, Discrete Groups, Expanding Graphs and Invariant Measures, Progress in Mathematics, 125, Birkhäuser, 1994. MR1308046 (96g:22018) 
[9] V. Pestov, Hyperlinear and sofic groups: a brief guide, Bull. Symbolic Logic 14 (2008), 449-480. MR2460675 (2009k:20103)

[10] T. Tsuzuku, Finite Groups and Finite Geometries, Cambridge Tracts in Mathematics, 78, Cambridge University Press, 1982. MR645725 (83b:20001)

Department of Mathematics, Rutgers University, 110 Frelinghuysen Road, PiscatAWAY, NEW JERSEY 08854-8019

E-mail address: sthomas@math.rutgers.edu 